\title{
Strangeness production within Parton-Hadron-String Dynamics (PHSD)
}

\author{
O. Linnyk ${ }^{a}$, E. L. Bratkovskaya ${ }^{a}$, W. Cassing ${ }^{b}$ \\ ${ }^{a}$ Institut für Theoretische Physik, Universität Frankfurt, 60438 Frankfurt am \\ Main, Germany \\ ${ }^{\mathrm{b}}$ Institut für Theoretische Physik, Universität Giessen, 35392 Giessen, Germany
}

\begin{abstract}
The Parton-Hadron-String Dynamics (PHSD) transport approach consistently simulates the full evolution of a relativistic heavy-ion collision from the initial hard scatterings string formation through the dynamical deconfinement phase transition to the quark gluon plasma (QGP), to the hadronization and to subsequent interactions in the hadronic phase. The transport theoretical description of quarks and gluons is based on a dynamical quasiparticle model for partons matched to reproduce recent lattice QCD results in thermodynamic equilibrium. The transition from partonic to hadronic degrees of freedom is described by covariant transition rates for the fusion of quark-antiquark pairs or three quarks (antiquarks). Studying $\mathrm{Pb}+\mathrm{Pb}$ reactions from 40 to $158 \mathrm{~A} \cdot \mathrm{GeV}$, we find that at most $40 \%$ of the collision energy is stored in the dynamics of the partons. This implies that a large fraction of non-partonic, i.e. hadronic or string-like matter, which can be viewed as a hadronic corona, is present in these reactions, thus neither hadronic nor purely partonic models can be employed to extract physical conclusions in comparing model results with data. On the other hand, comparing the PHSD results to those of the Hadron-String Dynamics (HSD) approach without the phase transition to QGP, we observe that the existence of the partonic phase has a sizeable influence on the transverse mass distribution of final kaons due to the repulsive partonic mean fields and initial partonic scattering. Furthermore, we find a significant effect of the QGP on the production of multi-strange antibaryons due to a slightly enhanced $s \bar{s}$ pair production in the partonic phase from massive time-like gluon decay and to a more abundant formation of strange antibaryons in the hadronization process.
\end{abstract}

Key words: Quark-gluon plasma, Relativistic heavy-ion collisions, Strangeness PACS: 12.38.Mh, 12.38.Aw, 25.75.-q

The nature of confinement and the phase transition from a partonic system of quarks, antiquarks and gluons - a quark-gluon plasma (QGP) - to interacting hadrons, as occurring in relativistic nucleus-nucleus collisions, is a central topic of modern 
high energy physics. In the present work, the dynamical evolution of the heavyion collision is described by the PHSD transport approach [1] incorporating the off-shell propagation of the partonic quasi-particles according to Ref. [2] as well as the transition to resonant hadronic states (or strings). Here we employ the PHSD approach - described in [1,3] - to strangeness production in nucleus-nucleus collisions at moderate relativistic energies, i.e. at SPS energies up to $160 \mathrm{~A} \cdot \mathrm{GeV}$.

\section{The PHSD approach}

A consistent dynamical approach - valid also for strongly interacting systems - can be formulated on the basis of the Kadanoff-Baym equations [4,5] or off-shell transport equations in phase-space representation, respectively [2,5]. In the Kadanoff-Baym theory the field quanta are described in terms of propagators with complex selfenergies. Whereas the real part of the selfenergies can be related to mean-field potentials, the imaginary parts provide information about the lifetime and/or reaction rates of time-like 'particles' [6]. Once the proper (complex) selfenergies of the degrees of freedom are known, the time evolution of the system is fully governed by off-shell transport equations (as described in Refs. [2,5]).

The PHSD approach is a microscopic covariant transport model that incorporates effective partonic as well as hadronic degrees of freedom and involves a dynamical description of the hadronization process from partonic to hadronic matter [1]. Whereas the hadronic part is essentially equivalent to the conventional HSD approach [7] the partonic dynamics is based on the Dynamical QuasiParticle Model (DQPM) 8] which describes QCD properties in terms of single-particle Green's functions (in the sense of a two-particle irreducible approach) and leads to effective strongly interacting partonic quasiparticles with broad spectral functions as degrees of freedom.

The off-shell parton dynamics also allows for a solution of the hadronization problem: the hadronization occurs by quark-antiquark fusion or 3 quark/3 antiquark recombination which is described by covariant transition rates as introduced in Ref. [1,3], obeying flavor current-conservation, color neutrality as well as energy-momentum conservation. Since the dynamical quarks become very massive close to $T_{c}$, the formed resonant 'pre-hadronic' color-dipole states $(q \bar{q}$ or $q q q)$ are of high invariant mass, too, and sequentially decay to the ground state meson and baryon octets increasing the total entropy. This solves the entropy problem in hadronization in a natural way [10].

\section{Partonic energy fractions}

We start with a consideration of energy partitions in order to map out the fraction of partonic energy in time for relativistic nucleus-nucleus collisions. The question emerges to what extent the partonic phase shows up as a function of centrality. To this aim we study the partonic energy $E_{p}(b)$ relative to the energy converted from the kinetic motion of the impinging nuclei at impact parameter $b$, i.e. $R_{p}(b)=E_{p}(b) /\left(E_{B}(t=0, b)-E_{B}(t \rightarrow \infty, b)\right)$, which is displayed in Fig. 1 for impact parameter $b$ from $1 \mathrm{fm}$ to $13 \mathrm{fm}$ in steps of $2 \mathrm{fm}$. The system is $\mathrm{Pb}+\mathrm{Pb}$ at 


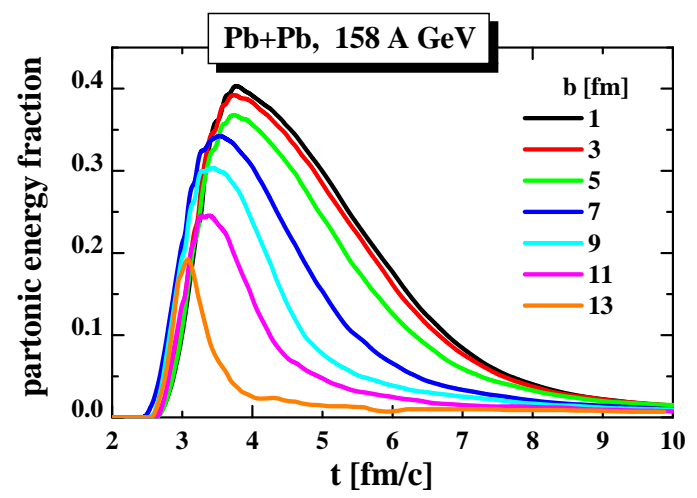

Fig. 1. The partonic energy fraction as a function of time for impact parameters $b$ from $1 \mathrm{fm}$ to $13 \mathrm{fm}$ in steps of $2 \mathrm{fm}$ for $\mathrm{Pb}+\mathrm{Pb}$ at $158 \mathrm{~A} \cdot \mathrm{GeV}$.

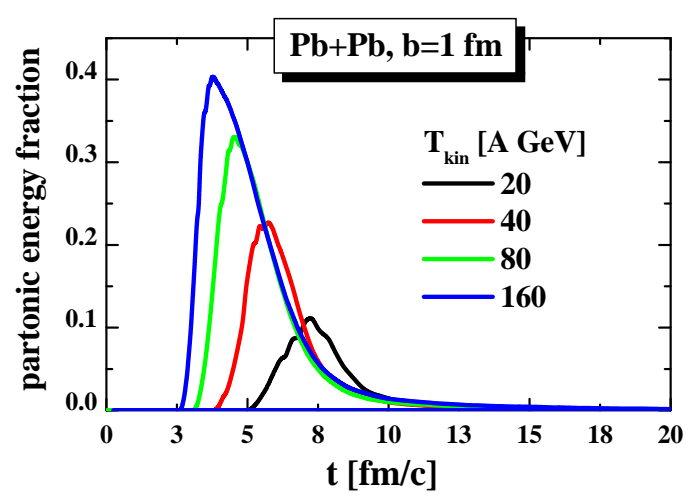

Fig. 2. The partonic energy fraction as a function of time for impact parameter $b=1 \mathrm{fm}$ for $\mathrm{Pb}+\mathrm{Pb}$ at $160,80,40$ and $20 \mathrm{~A} \cdot \mathrm{GeV}$.

$158 \mathrm{~A} \cdot \mathrm{GeV}$. One observes that even at very central collisions the partonic energy fraction $R_{p}$ only reaches about $40 \%$ and decreases to about $20 \%$ (in the peak) for very peripheral reactions. More striking is the fact that the duration of the partonic phase shrinks substantially when going from central to peripheral reactions. Thus the 'popular picture' that a partonic phase is reached in the overlap region of the nuclei at top SPS energies is by far not substantiated by the PHSD calculations. This has been been pointed out before in Refs. [11. In conclusion, very central collisions show a sizeable fraction of space-time regions of partonic nature, but also of the hadronic (or string-like) corona. At first sight this looks discouraging, but one should concentrate on observables with a special sensitivity to the partonic phase, e.g. strangeness [3], dileptons [12], charm [13] or jets [14].

We continue with the ratio $R_{p}$ as a function of bombarding energy concentrating here on the FAIR and full SPS energy regime but considering only central collisions $(b=1 \mathrm{fm})$. The results are displayed in Fig. 2 for central $\mathrm{Pb}+\mathrm{Pb}$ collisions at $160,80,40$, and $20 \mathrm{~A} \cdot \mathrm{GeV}$ as a function of time and demonstrate that the average duration of the partonic phase does not change very much with bombarding energy, however, the partonic volume shrinks by about a factor of three when stepping down in bombarding energy from 160 to $20 \mathrm{~A} \cdot \mathrm{GeV}$. Thus according to our PHSD calculations there should be a QGP also at FAIR energies but its space-time volume is significantly smaller than that for the hadronic phase.

\section{Particle spectra in comparison to experiment}

It is of interest, how the PHSD approach compares to the HSD [7] model (without explicit partonic degrees of freedom) as well as to experimental data. In Fig. 3 we show the transverse mass spectra of $\pi^{-}, K^{+}$and $K^{-}$mesons for $7 \%$ central $\mathrm{Pb}+\mathrm{Pb}$ collisions at 40 and $80 \mathrm{~A} \cdot \mathrm{GeV}$ and $5 \%$ central collisions at $158 \mathrm{~A} \cdot \mathrm{GeV}$ in comparison to the data of the NA49 Collaboration [15]. Here the slope of the $\pi^{-}$spectra is only slightly enhanced in PHSD relative to HSD which demonstrates that the pion transverse motion shows no sizeable sensitivity to the partonic phase. However, the 

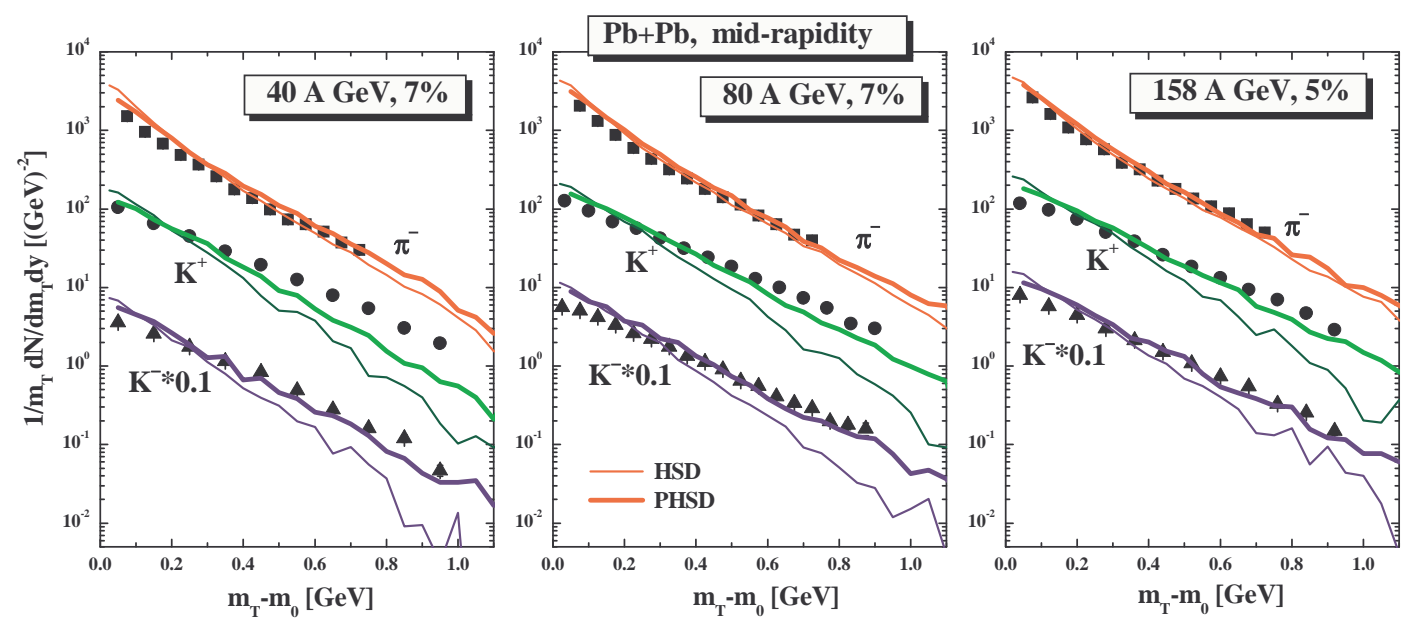

Fig. 3. The $\pi^{-}, K^{+}$and $K^{-}$transverse mass spectra for central $\mathrm{Pb}+\mathrm{Pb}$ collisions at 40 , 80 and $158 \mathrm{~A} \cdot \mathrm{GeV}$ from PHSD (thick solid lines) in comparison to the distributions from HSD (thin solid lines) and the experimental data from the NA49 Collaboration [15].

$K^{ \pm}$transverse mass spectra are substantially hardened with respect to the HSD calculations at all bombarding energies - i.e. PHSD is more in line with the data and thus suggest that partonic effects are better visible in the strangeness-degrees of freedom. The hardening of the kaon spectra can be traced back to initial partonparton scattering as well as a larger collective acceleration of the partons in the transverse direction due to the presence of repulsive vector fields for the partons. We recall that in Refs. [16] the underestimation of the $K^{ \pm}$slope by HSD (and also UrQMD) had been suggested to be a signature for missing partonic degrees of freedom; our present PHSD calculations support this early suggestion.

The strange antibaryon sector is of further interest since here the HSD calculations have always underestimated the yield [17]. In this respect we compare in Fig. 4 (top part) the multiplicities of $\left(\Lambda+\Sigma^{0}\right) / N_{\text {wound }}$ (l.h.s.) and $\left(\bar{\Lambda}+\bar{\Sigma}^{0}\right) / N_{\text {wound }}$ (r.h.s.) as functions of the number of wounded nucleons $N_{\text {wound }}$ for $\mathrm{Pb}+\mathrm{Pb}$ collisions at $158 \mathrm{~A} \cdot \mathrm{GeV}$ at mid-rapidity from PHSD and HSD to the experimental data from the NA57 Collaboration [18] and the NA49 Collaboration [19]. Whereas the HSD and PHSD calculations both give a reasonable description of the $\Lambda+\Sigma^{0}$ yield of the NA49 Collaboration, both models underestimate the NA57 data (open triangles) by about 30\%. An even larger discrepancy in the data from the NA49 and NA57 Collaborations is seen for $\left(\bar{\Lambda}+\bar{\Sigma}^{0}\right) / N_{\text {wound }}$ (r.h.s.); here the PHSD calculations give results which are in between the NA49 data (solid dots) and the NA57 data (open triangles). We see that HSD underestimates the $\left(\bar{\Lambda}+\bar{\Sigma}^{0}\right)$ midrapidity yield at all centralities. This observation points towards a partonic origin but needs further examination.

The latter result suggests that the partonic phase does not show up explicitly in an enhanced production of strangeness (or in particular strange mesons and baryons) but leads to a different redistribution of antistrange quarks between mesons and antibaryons. To examine this issue we show in Fig. 4 (bottom part) the multiplicities of $\Xi^{-}$baryons (l.h.s.) and $\bar{\Xi}^{+}$antibaryons (r.h.s.) vs. $N_{\text {wound }}$ for $\mathrm{Pb}+\mathrm{Pb}$ collisions at 

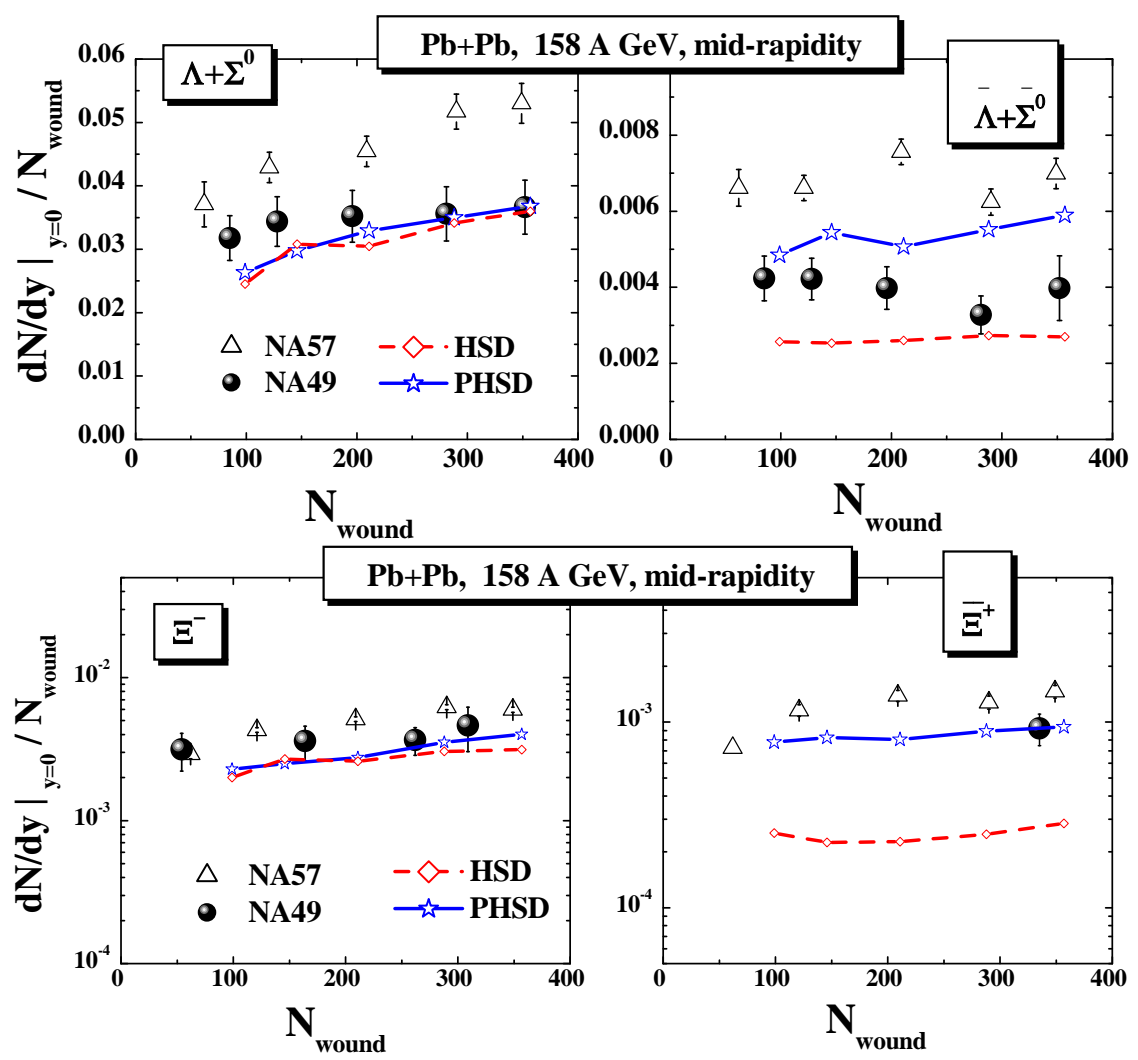

Fig. 4. Top: The multiplicities of $\left(\Lambda+\Sigma^{0}\right) / N_{\text {wound }}$ (l.h.s.) and $\left(\bar{\Lambda}+\bar{\Sigma}^{0}\right) / N_{\text {wound }}$ (r.h.s.) as a function of the number of wounded nucleons for $\mathrm{Pb}+\mathrm{Pb}$ collisions at $158 \mathrm{~A} \cdot \mathrm{GeV}$ at mid-rapidity from PHSD (blue solid lines) and HSD (red dashed-dotted lines) in comparison to the experimental data from the NA57 Collaboration [18] (open triangles) and the NA49 Collaboration [19] (solid dots). The calculations have an error of $5-10 \%$ due to limited statistics. Bottom: The multiplicities of $\Xi^{-} / N_{\text {wound }}$ (l.h.s.) and $\bar{\Xi}^{+} / N_{\text {wound }}$ (r.h.s.) vs $N_{\text {wound }}$ for $\mathrm{Pb}+\mathrm{Pb}$ collisions at $158 \mathrm{~A} \cdot \mathrm{GeV}$ at mid-rapidity. Line coding as above.

$158 \mathrm{~A} \cdot \mathrm{GeV}$ at mid-rapidity from PHSD and HSD in comparison to the experimental data from the NA57 Collaboration [18] and the NA49 Collaboration [20,19]. The situation is very similar to the case of the strange baryons and antibaryons before: we find no sizeable differences in the double strange baryons from HSD and PHSD in a good agreement with the NA49 data - but observe a large enhancement in the double strange antibaryons for PHSD relative to HSD.

\section{Summary}

The PHSD approach has been applied to nucleus-nucleus collisions from 20 to $160 \mathrm{~A} \cdot \mathrm{GeV}$ in order to explore the space-time regions of 'partonic matter'. We have found that even central collisions at the top SPS energy of $\sim 160 \mathrm{~A} \cdot \mathrm{GeV}$ show a large fraction of non-partonic matter. It is also found that though the partonic phase has only a very low impact on rapidity distributions of hadrons [3], it has a sizeable influence on the transverse-mass distribution of final kaons due to the repulsive partonic mean fields and initial parton interactions. On the other hand, the most pronounced effect of the partonic phase is seen on the production of multi-strange antibaryons 
due to a slightly enhanced $s \bar{s}$ pair production in the partonic phase from massive time-like gluon decay and a more abundant formation of strange antibaryons in the hadronization process. We also mention that partonic production channels for dilep-

tons appear to be visible in the $\mu^{+} \mu^{-}$spectra from In+In collisions at $158 \mathrm{~A} \cdot \mathrm{GeV}$ in the intermediate invariant mass range [12].

Supported in part by the "HIC for FAIR" center of the "LOEWE" program.

\section{References}

[1] W. Cassing and E. L. Bratkovskaya, Phys. Rev. C 78 (2008) 034919.

[2] W. Cassing and S. Juchem, Nucl. Phys. A 665 (2000) 377; ibid A 672 (2000) 417.

[3] W. Cassing and E. L. Bratkovskaya, Nucl. Phys. A 831 (2009) 215.

[4] L. P. Kadanoff, G. Baym, Quantum Statistical Mechanics, Benjamin, 1962.

[5] W. Cassing and S. Juchem, Nucl. Phys. A 672 (2000) 417; S. Juchem et al., Nucl. Phys. A 743200492.

[6] A. Peshier and W. Cassing, Phys. Rev. Lett. 94 (2005) 172301.

[7] W. Cassing and E. L. Bratkovskaya, Phys. Rept. 308 (1999) 65; W. Ehehalt and W. Cassing, Nucl. Phys. A 602 (1996) 449.

[8] W. Cassing, Nucl. Phys. A 791 (2007) 365; ibid. A 795 (2007) 70.

[9] W. Cassing and E. L. Bratkovskaya, Phys. Rev. C 78 (2008) 034919.

[10] W. Cassing, E. L. Bratkovskaya and Y. Xing, Prog. Part. Nucl. Phys. 62 (2009) 359.

[11] J. Aichelin and K. Werner, Phys. Rev. C 79, 064907 (2009); K. Werner, Phys. Rev. Lett. 98 (2007) 152301; F. Beccattini and J. Manninen, J. Phys. G 35 (2008) 104013.

[12] O. Linnyk, E. L. Bratkovskaya and W. Cassing, Nucl. Phys. A 830 (2009) 491C.

[13] O. Linnyk, E. L. Bratkovskaya and W. Cassing, Int. J. Mod. Phys. E 17 (2008) 1367.

[14] K. Gallmeister and W. Cassing, Nucl. Phys. A 748 (2005) 241.

[15] C. Alt et al., NA49 Collaboration, Phys. Rev. C 66 (2002) 054902; Phys. Rev. C 77 (2008) 024903.

[16] E. L. Bratkovskaya, S. Soff, H. Stöcker, M. van Leeuwen, and W. Cassing, Phys. Rev. Lett. 92 (2004) 032302; E. L. Bratkovskaya, W. Cassing, and H. Stöcker, Phys. Rev. C 67 (2003) 054905; E. L. Bratkovskaya et al., Phys. Rev. C 69 (2004) 054907.

[17] J. Geiss, W. Cassing and C. Greiner, Nucl. Phys. A 644 (1998) 107.

[18] F. Antinori et al., Phys. Lett. B 595 (2004) 68; J. Phys. G: Nucl. Phys. 32 (2006) 427.

[19] T. Anticic et al. [NA49 Collaboration], Phys. Rev. C 80 (2009) 034906.

[20] C. Alt et al., NA49 Collaboration, Phys. Rev. C 78 (2008) 034918.

[21] J. Rafelski and B. Müller, Phys. Rev. Lett. 48 (1982) 1066. 